\title{
Phase-only shaped laser pulses in optimal control theory: Application to indirect photofragmentation dynamics in the weak-field limit
}

Shu, Chuan-Cun; Henriksen, Niels E.

Published in:

Journal of Chemical Physics

Link to article, DOI:

$10.1063 / 1.3678013$

Publication date:

2012

Document Version

Publisher's PDF, also known as Version of record

Link back to DTU Orbit

Citation (APA):

Shu, C-C., \& Henriksen, N. E. (2012). Phase-only shaped laser pulses in optimal control theory: Application to indirect photofragmentation dynamics in the weak-field limit. Journal of Chemical Physics, 136(4), 044303. https://doi.org/10.1063/1.3678013

\section{General rights}

Copyright and moral rights for the publications made accessible in the public portal are retained by the authors and/or other copyright owners and it is a condition of accessing publications that users recognise and abide by the legal requirements associated with these rights.

- Users may download and print one copy of any publication from the public portal for the purpose of private study or research.

- You may not further distribute the material or use it for any profit-making activity or commercial gain

- You may freely distribute the URL identifying the publication in the public portal 


\section{AIP $\begin{gathered}\text { molowanat } \\ \text { chemical Physics }\end{gathered}$}

Phase-only shaped laser pulses in optimal control theory: Application to indirect photofragmentation dynamics in the weak-field limit

Chuan-Cun Shu and Niels E. Henriksen

Citation: J. Chem. Phys. 136, 044303 (2012); doi: 10.1063/1.3678013

View online: http://dx.doi.org/10.1063/1.3678013

View Table of Contents: http://jcp.aip.org/resource/1/JCPSA6/v136/i4

Published by the American Institute of Physics.

Additional information on J. Chem. Phys.

Journal Homepage: http://jcp.aip.org/

Journal Information: http://jcp.aip.org/about/about_the_journal

Top downloads: http://jcp.aip.org/features/most_downloaded

Information for Authors: http://jcp.aip.org/authors

Explore AIP's new open-access journal

Article-level metrics now available

Join the conversation! Rate $\&$ comment on articles 


\title{
Phase-only shaped laser pulses in optimal control theory: Application to indirect photofragmentation dynamics in the weak-field limit
}

\author{
Chuan-Cun Shu and Niels E. Henriksen a) \\ Department of Chemistry, Building 207, Technical University of Denmark, DK-2800, \\ Kongens Lyngby, Denmark
}

(Received 2 November 2011; accepted 2 January 2012; published online 23 January 2012)

\begin{abstract}
We implement phase-only shaped laser pulses within quantum optimal control theory for lasermolecule interaction. This approach is applied to the indirect photofragmentation dynamics of $\mathrm{NaI}$ in the weak-field limit. It is shown that optimized phase-modulated pulses with a fixed frequency distribution can substantially modify transient dissociation probabilities as well as the momentum distribution associated with the relative motion of Na and I. (C) 2012 American Institute of Physics. [doi:10.1063/1.3678013]
\end{abstract}

\section{INTRODUCTION}

Within the last decade it has become possible to tailor the electric field of laser pulses

$$
\mathcal{E}(t)=\operatorname{Re}\left[\int_{-\infty}^{\infty} E(\omega) e^{i \phi(\omega)} e^{-i \omega t} d \omega\right],
$$

via the control of the spectral amplitudes of the frequency distribution $E(\omega)$ as well as the associated phases $\phi(\omega)$ (see, e.g., Ref. 1). In laser-molecule interaction, the control of these macroscopic parameters in the laboratory leads to control at the molecular level. For example, the variation of the phases implies that quantum interferences, associated with the coherent excitation of quantum states, are controlled.

The theoretical study of laser control has also progressed both in terms of insights and methods, see, e.g., Refs. 2-4. The most effective theoretical approach to design laser pulses in order to achieve a desired outcome of photophysical processes is based on the framework of optimal control theory (OCT) ${ }^{2,5}$ Usually, OCT will modify both the frequency distribution and the phases in order to find an optimized laser pulse.

Many experiments are carried out using only phase modulation of the laser pulses. This reduces the "search space" for optimizing the effect of the laser pulse. Furthermore, this approach has the advantage of conserving the pulse energy and hence highlighting the effect of quantum coherence phenomena. If a phase-only shaped approach is used there exists no unique way to determine the phase function that will lead to a desired outcome of the laser-molecule interaction. Usually optimization algorithms such as genetic algorithms have been employed to address this problem. Phase-only shaped laser pulses have been optimized by this approach combined with feedback from an experiment (see, e.g., Ref. 6) or from a theoretical description of the dynamics obtained from a numerical solution of the time-dependent Schrödinger equation (see, e.g., Ref. 7).

\footnotetext{
a)Electronic mail: neh@kemi.dtu.dk.
}

In this work, we present a first implementation and elaboration on an OCT scheme for phase-only shaping which is based on the approach sketched in Ref. 5 .

We focus on control of photofragmentation in the weakfield limit. With a fixed frequency distribution and hence pulse energy, coherent control in the weak-field (one-photon) limit exploits quantum interference in its purest form. ${ }^{3,8}$ However, when excitation out of a single eigenstate is considered and direct fragmentation takes place within a dissociative continuum of states, phase control of final state distributions of the fragments is impossible for closed systems, ${ }^{9-12}$ although the form of the nuclear wave packet can be controlled. ${ }^{13,14}$

Recently, we showed that for indirect photofragmentation in the weak-field limit, transient fragment distributions (such as the dissociation probability and momentum distribution) can be changed by a phase-modulated pulse with a fixed bandwidth. ${ }^{15}$ This result can be considered as a supplement to the one-photon no control result of Brumer and Shapiro. ${ }^{9}$ It applies only to transient fragment distributions, that is, no control is observed in the long-time limit of an indirect fragmentation such as $\mathrm{NaI} \rightarrow(\mathrm{NaI})^{*} \rightarrow \mathrm{Na}+\mathrm{I}$, when the quasibound complex $(\mathrm{NaI})^{*}$ has disappeared. In our previous work, we considered only a simple phase modulation leading to linearly chirped laser pulses. In this work, we will extend this study and optimize the effect of a phase-modulated pulse.

This paper is organized in the following way: Section II A presents the algorithm for phase-only pulse shaping within the framework of OCT. Section II B gives a brief summary of the theoretical description of the indirect laser-induced fragmentation of NaI. Numerical results and discussion are presented in Sec. III. Finally, conclusions are given in Sec. IV.

\section{THEORETICAL APPROACH}

\section{A. Phase-only pulse shaping}

Within the electric-dipole approximation, the Hamiltonian for the interaction between a system and a planepolarized electric field takes the form

$$
\hat{H}(t)=\hat{H}_{0}-\hat{\mu} \mathcal{E}(t)
$$


where $\hat{H}_{0}$ is the Hamiltonian of the field-free system, $\hat{\mu}$ is the projection of the dipole moment operator on the polarization vector of the field, and $\mathcal{E}(t)$ is the electric field. The time evolution of the system is given by the time-dependent Schrödinger equation ( $\hbar \equiv 1$ in the following)

$$
i \frac{\partial}{\partial t}|\psi(t)\rangle=\hat{H}(t)|\psi(t)\rangle, \quad|\psi(0)\rangle=\left|\phi_{i}\right\rangle .
$$

Now the aim is to find the electric field $\mathcal{E}(t)$ which drives the system from its initial state $|\psi(0)\rangle=\left|\phi_{i}\right\rangle$ to a state $|\psi(T)\rangle$, which is as close as possible to a desired target state $\left|\phi_{f}\right\rangle$.

By taking into account constraints on the external field, optimal control can be formulated as the problem of maximizing an objective functional $J$ of the electric field, ${ }^{5,16}$ where

$$
J=J_{S}-\alpha \int_{0}^{T} d t \frac{|\mathcal{E}(t)|^{2}}{s(t)},
$$

with the system dependent part

$$
\begin{aligned}
J_{S}= & \left|\left\langle\psi(T) \mid \phi_{f}\right\rangle\right|^{2}-2 \operatorname{Re}\left\{\left\langle\psi(T) \mid \phi_{f}\right\rangle\right. \\
& \left.\times \int_{0}^{T} d t\left\langle\chi(t)\left|\left(i \hat{H}(t)+\frac{\partial}{\partial t}\right)\right| \psi(t)\right\rangle\right\} .
\end{aligned}
$$

On the right-hand side of Eq. (5), the first term is the original control objective, the second term is to ensure that the time evolution follows the Schrödinger equation, Eq. (3), and $|\chi\rangle$ is the so-called Lagrange multiplier. The second term in Eq. (4) represents constraints on the control field. $\alpha$ is a constant positive Lagrange multiplier chosen to weight the significance of the pulse energy, and $s(t)$ is a shape function which enforces a smooth switch on and off of the field.

It can be shown that $J$ is maximized when the following equations are satisfied ${ }^{5,16}$ :

$$
i \frac{\partial}{\partial t}|\chi(t)\rangle=\hat{H}(t)|\chi(t)\rangle, \quad|\chi(T)\rangle=\left|\phi_{f}\right\rangle,
$$

where the real-valued control field is obtained from the real part of the expression

$$
\mathcal{E}_{c}(t)=i \frac{s(t)}{\alpha}\langle\psi(t) \mid \chi(t)\rangle\langle\chi(t)|\hat{\mu}| \psi(t)\rangle .
$$

The system of coupled equations, Eqs. (3), (6), and (7) are solved using an iterative method. ${ }^{5}$ Due to the initial conditions, Eqs. (3) and (6) correspond to a forward and a backward propagation in time, respectively. The design of the optimal pulse requires typically an intensive iteration process to modify the initial optical pulse to achieve the desired objective.

In iterations associated with the calculation of $\chi(t)$, the electric field used for calculating the backward-propagating wave function is taken from the real part of the control field in Eq. (7), i.e., $\mathcal{E}^{\prime}(t)=\operatorname{Re}\left[\mathcal{E}_{c}^{\prime}(t)\right]$. For the implementation of phase-only shaping into the computational optimization, we have to decode the phase information from the field in Eq. (7). First, the Fourier transformation of the pulse to the spectral domain is performed by

$$
E^{\prime}(\omega)=\frac{1}{2 \pi} \int_{-\infty}^{\infty} \mathcal{E}_{c}^{\prime}(t) e^{i \omega t} d t,
$$

where $E^{\prime}(\omega)$ is a complex function containing all the information concerning the pulse and from which the complex $\mathcal{E}_{c}^{\prime}(t)$ in Eq. (7) can be retrieved. Then the electric field $\mathcal{E}(t)$ in the time domain for calculating the forward-propagating wave function $\psi(t)$ can be obtained by

$$
\begin{aligned}
\mathcal{E}(t) & =\frac{1}{2 \pi} \operatorname{Re}\left[\int_{-\infty}^{\infty} E_{i n}(\omega) e^{i \phi(\omega)} e^{-i \omega t} d \omega\right] \\
& =\frac{1}{2 \pi} \operatorname{Re}\left[\int_{-\infty}^{\infty} E_{i n}(\omega) \frac{E^{\prime}(\omega)}{\left|E^{\prime}(\omega)\right|} e^{-i \omega t} d \omega\right],
\end{aligned}
$$

where $E_{\text {in }}(\omega)$ is the predefined spectral distribution, which in an experiment corresponds to the spectrum of laser pulse that enters the pulse-shaping device. This phase-only shaping scheme is similar to that sketched in Ref. 5, but is presented here in a more detailed way. We assume that the optimized phase-shaped pulse is generated from a Fourier transform limited (FTL) pulse that can be obtained by conventional laser systems. The frequency distribution is therefore given by a Gaussian

$$
E_{\text {in }}(\omega)=E_{0} \exp \left[-\ln 2\left(\frac{\omega-\omega_{0}}{\Delta \omega / 2}\right)^{2}\right],
$$

where $E_{0}, \omega, \omega_{0}$, and $\Delta \omega$ are the peak field strength, the angular frequency, the center frequency, and the bandwidth (FWHM) of the laser pulse, respectively.

For later reference, we note that to analyze the effect of phase, the spectral phase $\phi(\omega)$ in Eq. (9) is usually expanded into a Taylor series around the center frequency $\omega_{0}$

$$
\begin{aligned}
\phi(\omega)= & \phi_{0}+\left(\omega-\omega_{0}\right) \phi_{1}+\frac{1}{2}\left(\omega-\omega_{0}\right)^{2} \phi_{2} \\
& +\frac{1}{6}\left(\omega-\omega_{0}\right)^{3} \phi_{3}+\cdots,
\end{aligned}
$$

where $\phi_{k}(k=0,1,3, \ldots)$ denotes the expansion coefficient of order $k$.

\section{B. Quantum dynamic description of $\mathrm{Nal} \rightarrow \mathrm{Na}+\mathrm{I}$}

We consider here the laser-induced fragmentation of a diatomic molecule, $\mathrm{NaI} \rightarrow \mathrm{Na}+\mathrm{I}$ via a quasi-bound $(\mathrm{NaI})^{*}$ complex, due to a curve crossing between a bound and a repulsive electronic state, see Fig. 1, where the relevant potential energy curves are from Ref. 17 (see also Ref. 18 and references therein). In the diabatic representation, the molecular Hamiltonian is expressed as

$$
\begin{aligned}
\hat{H}_{0}(R)= & \sum_{n=i, c} \int d R|n R\rangle\left[\hat{T}+\hat{V}_{n n}(R)\right]\langle n R| \\
& +\int d R\left(|i R\rangle \hat{V}_{i c}(R)\langle c R|+| c R\rangle \hat{V}_{c i}(R)\langle i R|\right),
\end{aligned}
$$

where $|i R\rangle=|i(R)\rangle|R\rangle(|c R\rangle=|c(R)\rangle|R\rangle)$ denotes the ionic (covalent) state with eigenstate of the nuclear coordinate, $|R\rangle$. The operators $\hat{T}, \hat{V}_{i i}(R)\left(\hat{V}_{c c}(R)\right)$, and $\hat{V}_{i c}(R)$ represent the kinetic energy operator, the diabatic potential energy of the ionic (covalent) state, and the coupling between the two diabatic states, respectively. The transition dipole moment 


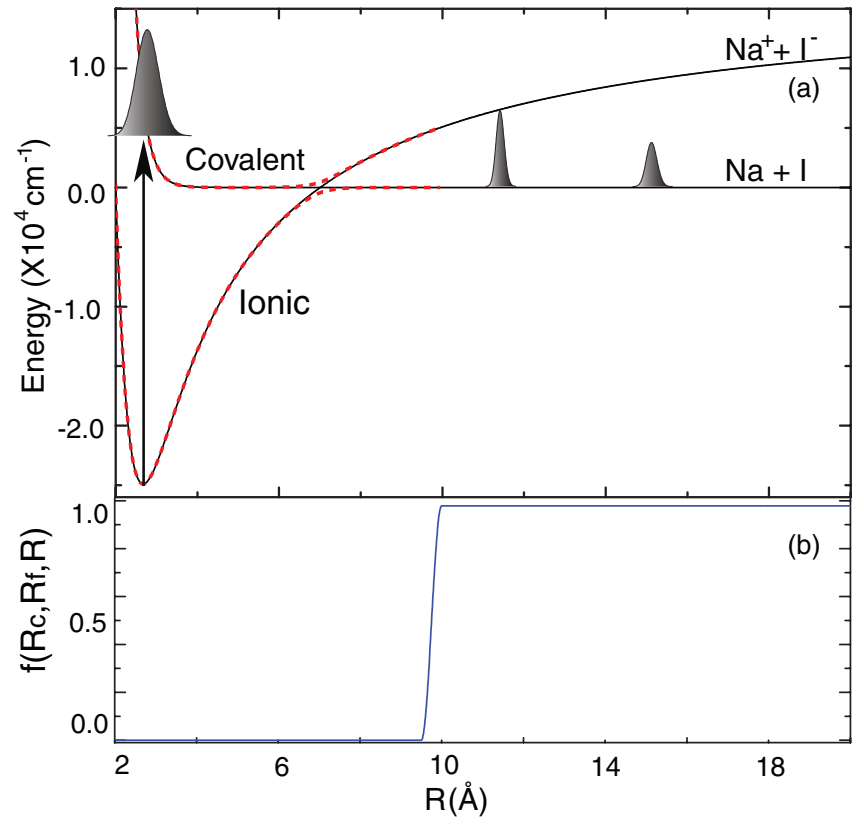

FIG. 1. Dissociation of NaI via non-adiabatic dynamics. (a) The dashed lines indicate the non-crossing adiabatic potentials. (b) The function $f\left(R_{c}, R_{f}, R\right)$ in the projection operator of Eq. (14).

function for the interaction with the field is given in Ref. 19, and the plane polarized field is assumed to be parallel to the transition dipole moment.

In the present study, we want to optimize the effect of a phase-modulated pulse on the probability of observing dissociation in the $\mathrm{Na}+\mathrm{I}$ channel. Under weak-field conditions, population transfer from the ground state to the excited state grows linearly with the intensity of the field, and is independent of phase. ${ }^{15,20} \mathrm{We}$ assumed that the population transferred to the excited state is $a^{2}$, and thus the target state can be defined by

$$
\left|\phi_{f}\right\rangle=\sqrt{1-a^{2}}|\psi(0)\rangle+a \frac{\hat{\mathrm{O}}_{\mathrm{c}}|\psi(T)\rangle}{\| \hat{\mathrm{O}}_{\mathrm{c}}|\psi(T)\rangle \|},
$$

where $|\psi(0)\rangle=\left|\phi_{i}\right\rangle$ is the initial vibrational ground state of $\mathrm{NaI}$. $\hat{O}_{c}$ is a projection operator that will effectively suppress the part of the wave packet in the coupling region and become a constant of 1 in the asymptotic region of the covalent state, where the wave packet can be regarded as representing dissociated fragments. Thus, it can be chosen as

$$
\hat{O}_{c}=\int_{0}^{\infty} d R|c R\rangle f\left(R_{c}, R_{f}, R\right)\langle c R|
$$

where

$$
f(x, y, z)= \begin{cases}0, & x<y, \\ \sin ^{2} \frac{\pi(x-y)}{2(z-y)}, & y \leq x \leq z \\ 1, & x>z,\end{cases}
$$

with $R_{c}=9.5 \AA$ and $R_{f}=10.0 \AA$. The function $f\left(R_{c}, R_{f}, R\right)$ is shown in Fig. 1(b).

The momentum distribution of the wave packet in the covalent state is computed by Fourier transforming the wave packet from position space to momentum space as

$$
\Phi(p, t)=\frac{1}{\sqrt{2 \pi}} \int_{-\infty}^{+\infty} e^{-i p R}\left\langle c R\left|\hat{O}_{c}\right| \psi(R, t)\right\rangle d R .
$$

It should be noted that the projection operator also represents the relevant Franck-Condon window function when the momentum distribution is probed via an ionization step. ${ }^{21}$ The details of the numerical method for solving the timedependent Schrödinger equation for this problem can be found in our previous paper. ${ }^{15}$

\section{RESULTS AND DISCUSSION}

As a test, the initial input pulse is a FTL pulse $(\phi(\omega)=0)$, centered at $t=0$, with full width at half maximum (FWHM) of $30 \mathrm{fs}$, and a center wavelength of $328 \mathrm{~nm}$. This is a typical pump wavelength used in experimental studies of NaI. ${ }^{17}$ The intensity is $1.0 \times 10^{11} \mathrm{~W} / \mathrm{cm}^{2}$, such that the calculations are performed in the weak-field regime ${ }^{15}\left(a^{2}=0.017\right.$ in Eq. (13)). The target time is set to $T=1000 \mathrm{fs}$, such that the wave packet always stayed within the grid region. In Eq. (4), $\alpha=1$ and the envelope function $s(t)$ of the optimized laser pulse is taken to be a Gaussian with center time at 0 fs and FWHM of $600 \mathrm{fs}$, that is, field-free conditions are established at about $500 \mathrm{fs}$ after the center of the pulse.

The optimized pulse $\mathcal{E}(t)$ in Eq. (9) is designed to transfer as much as possible of the population in the excited state into the channel $\mathrm{Na}+\mathrm{I}$, at the target time. When we apply the OCT algorithm, the main feature of the optimized pulse is a pulse centered at negative times, i.e., it is essentially a timeshifted version of the initial pulse. From the point of view of the representation of the phase function in Eq. (11), we note that according to the Fourier transform shift theorem, a linear term in the spectral phase $\phi_{1}$ leaves the laser field envelope unchanged, but shift the pulse in the time domain. This is a rather trivial solution to the problem.

In the present study, we want to find a symmetric pulse with center time at $t=0$, so that we can directly compare the results between shaped and unshaped pulses. Thus, we invoke the constraint of an even phase function $\phi\left(\omega-\omega_{0}\right)=\phi\left(\omega_{0}\right.$ $-\omega)$, which implies that the phase in Eq. (9) can be obtained by the following two approaches:

$$
\text { I : } \quad e^{i \phi(\omega)}= \begin{cases}\frac{E^{\prime}(\omega)}{\left|E^{\prime}(\omega)\right|}, & \omega \geq \omega_{0}, \\ \frac{E^{\prime}\left(2 \omega_{0}-\omega\right)}{\left|E^{\prime}\left(2 \omega_{0}-\omega\right)\right|}, & \omega<\omega_{0},\end{cases}
$$

or

$$
\text { II }: \quad e^{i \phi(\omega)}= \begin{cases}\frac{E^{\prime}(\omega)}{\left|E^{\prime}(\omega)\right|}, & \omega \leq \omega_{0}, \\ \frac{E^{\prime}\left(2 \omega_{0}-\omega\right)}{\left|E^{\prime}\left(2 \omega_{0}-\omega\right)\right|}, & \omega>\omega_{0},\end{cases}
$$

Figure 2 shows the control objective $\left\langle\psi(T)\left|\hat{O}_{c}\right| \psi(T)\right\rangle$ as a function of the number of iterations with the above phase functions. We find that the part of the wave packet which has moved into the asymptotic region under the constraint of constant pulse energy is significantly enhanced by appropriately modifying the spectral phase of a many-cycle femtosecond 


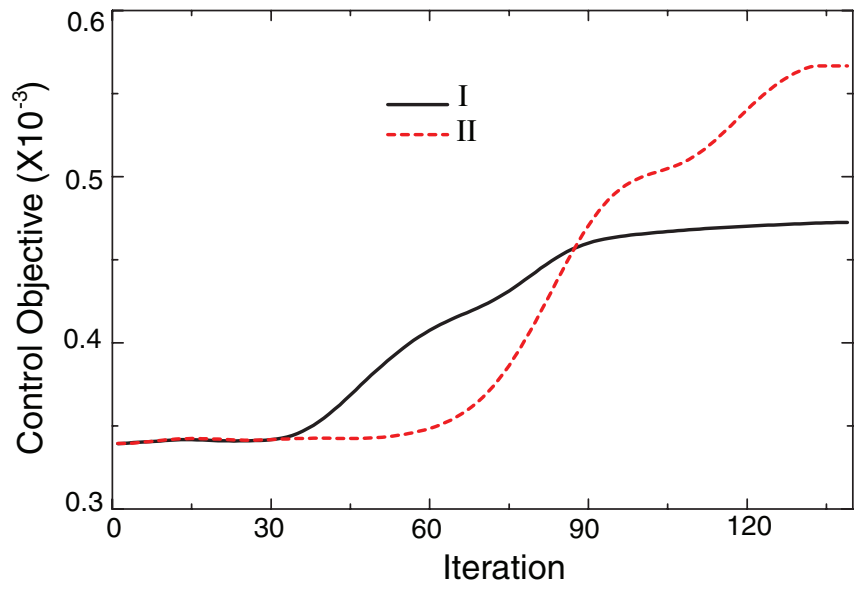

FIG. 2. The control objective $\left\langle\psi(T)\left|\hat{O}_{c}\right| \psi(T)\right\rangle$ as a function of the number of iterations with two different spectral phase functions; I from Eq. (17), and II from Eq. (18). The result at the 0th iteration corresponds to the initial unshaped transform-limited pulse.

laser pulse. Furthermore, the dissociation probability with the first kind of phase function is smaller than that with the second one. Attempts to minimize the control objective suggests that the transform-limited pulse gives the smallest dissociation probability.

Since, for phase-only shaping, we have to add constraints on the standard OCT algorithm, we cannot expect a monotonic convergence. In this case one can simply store the field which gives the highest yield in the memory, and this field is the result of the optimization. Although a proof of monotonic convergence similar to that in Ref. 22 is not possible here, as can be seen in Fig. 2, the obtained results show initially almost monotonic convergence. The control objective is maximized after about 130 iterations (more precisely 130 for pulse I and 135 iterations for pulse II). Subsequently, we observe that the control objective will decrease. At the maximum, the pulse has spread to the maximum temporal duration allowed by the envelope function $s(t)$ and in subsequent iterations, the electric field outside the envelope will be cutoff, leading to a reduced pulse energy.

The optimized laser pulses found by the OCT algorithm are shown in Fig. 3, where the electric fields are described by intensity and phase in the frequency and/or the time domain. The temporal pulse width (FWHM) was stretched to $600 \mathrm{fs}$ from the initial FWHM of 30 fs (see Figs. 3(a) and 3(e)) and the spectral intensities are, as expected, exactly unchanged after the pulse shaping (see Figs. 3(b) and 3(f)). The quadratic term in Eq. (11) leads to a linear chirp of the pulse in the time domain, which induces an increase of the pulse duration. It is very interesting to note that the phases and the time-frequency dependent representations show the main features of a linearly chirped laser pulse in the neighborhood of the center frequency $\omega_{0}$. The phases are given in the range $-\pi \leq \phi(\omega)$ $\leq \pi$, but it should be noted that $\exp [i \phi(\omega)]=\exp [i(\phi(\omega)+$ $n 2 \pi)$ ], where $n$ is an integer. The optimized pulse by the first spectral phase function mainly consists of a positive chirped pulse (see Fig. 3(c) and 3(d)), and the second one is similar to a negative chirped pulse (see Fig. 3(g) and 3(h)). Higher order phase modulation is, however, also observed.

In our previous work, ${ }^{15}$ we considered only linearly chirped laser pulses and obtained similar enhancements of the dissociation probability. However, it should be noted that the FWHM of these pulses (i.e., $740 \mathrm{fs}$ ) was larger than the 600 fs of the optimized pulses in Fig. 3. Furthermore, one could ask the question whether a result similar to that of the optimized pulse, could have been obtained simply by
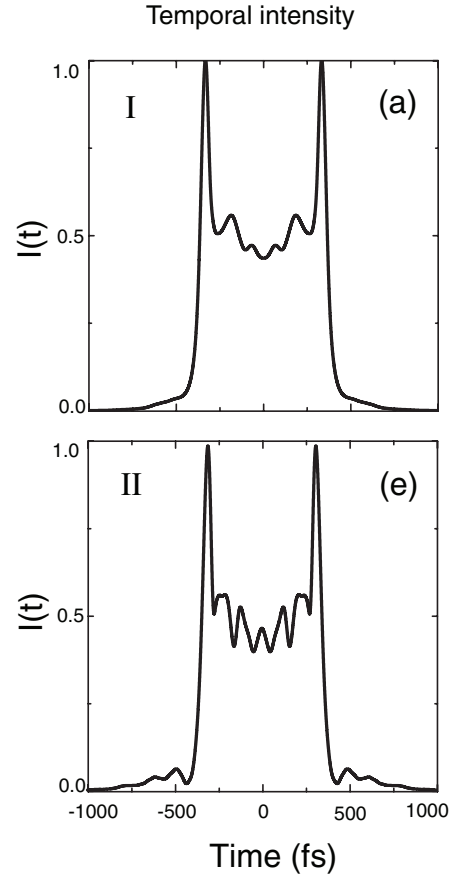

Spectral intensity
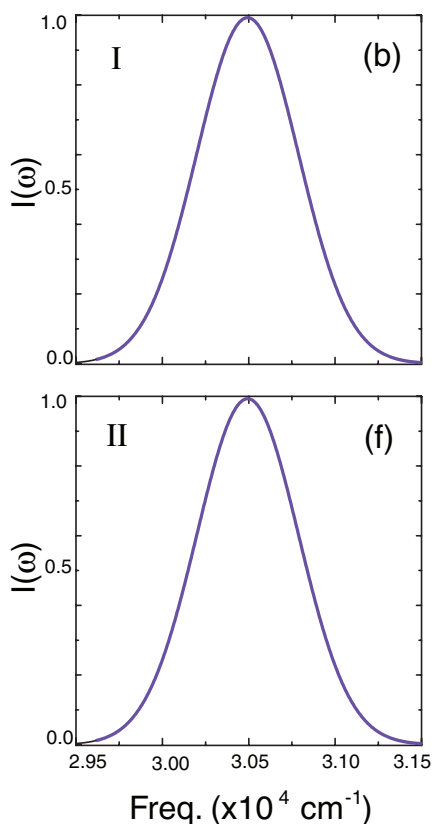

Spectral phase
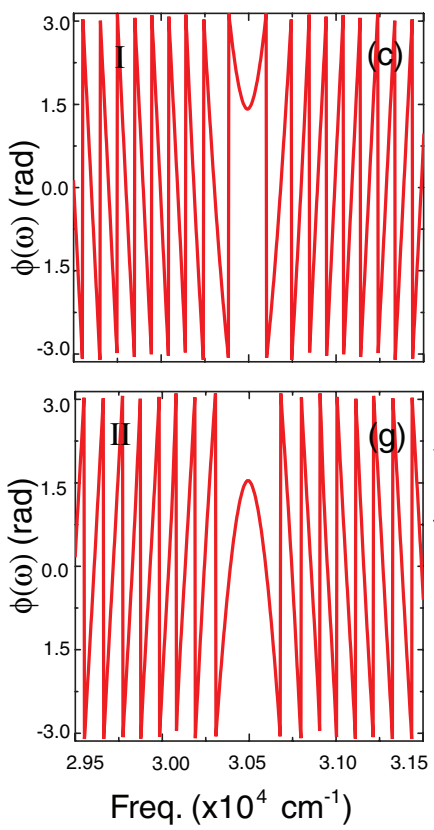

Spectrogram
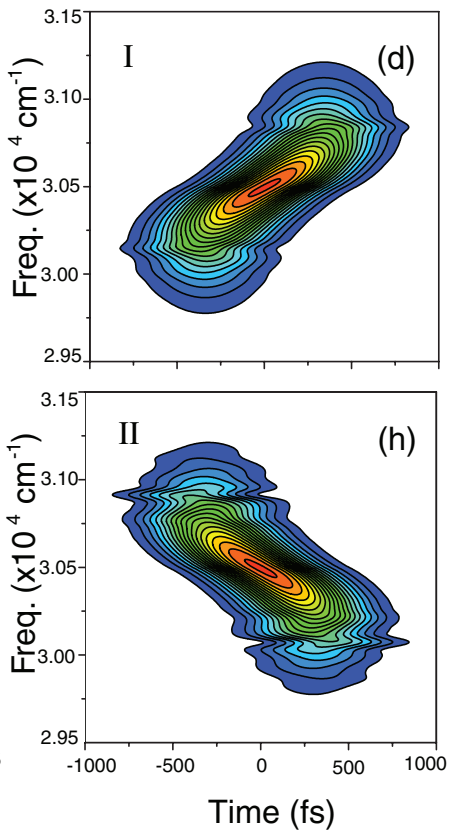

FIG. 3. Optimized laser pulses. The first column shows the temporal intensity, the second one the spectral intensity, the third one the phase, and the last one the Husimi transform power spectra as a function of time and frequency. (a)-(d) corresponds to phase function I of Eq. (17), and (e)-(h) corresponds to phase function II of Eq. (18). 

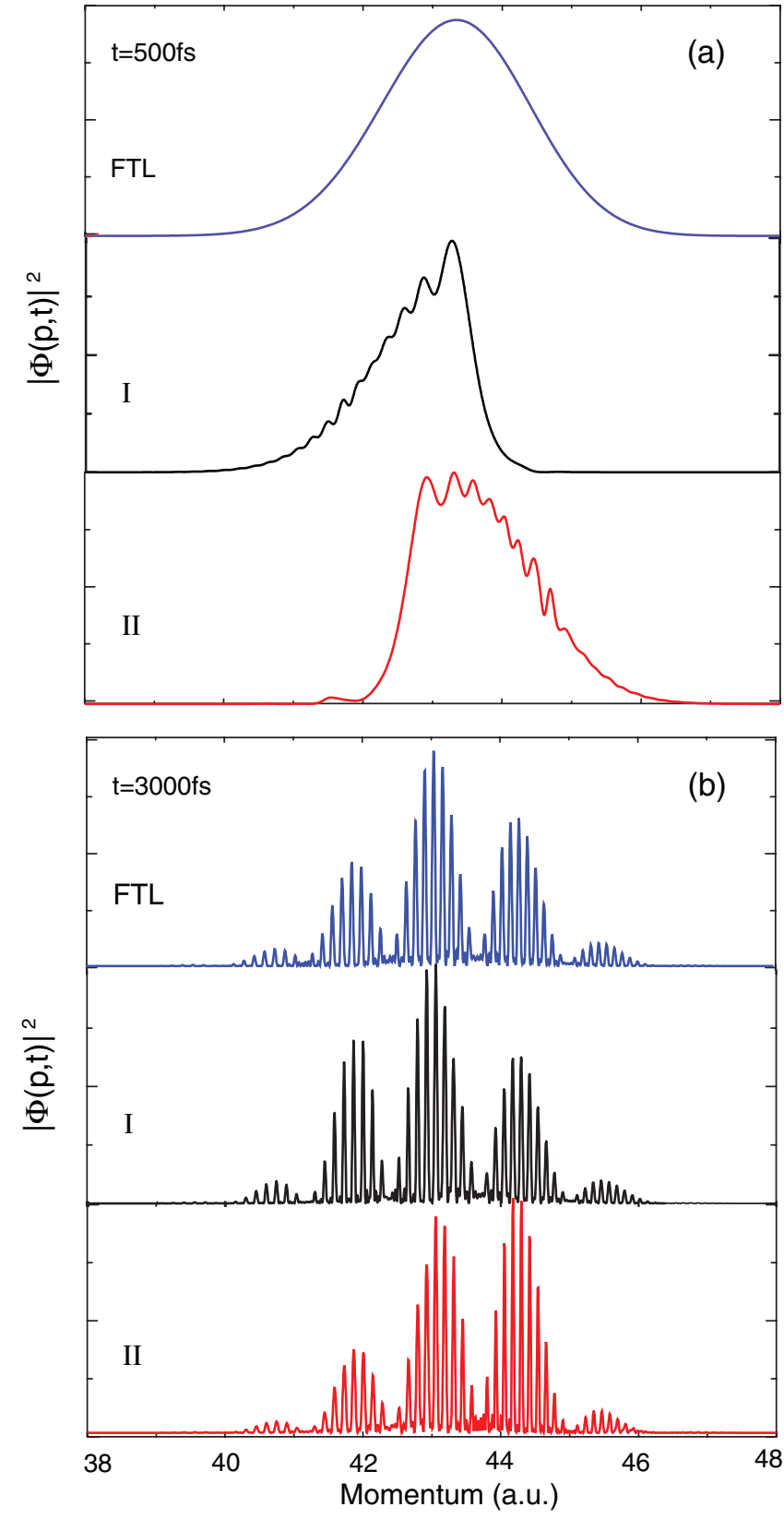

FIG. 4. The momentum distributions with two kinds of phase-only shaped pulses, I from Eq. (17), and II from Eq. (18), at $t=500$ and $t=3000 \mathrm{fs,}$ respectively. FTL denotes the corresponding Fourier transform-limited pulse.

choosing a different linear chirping rate. This is not the case, because with a pulse duration of $600 \mathrm{fs}$ (as for the optimized pulse), corresponding to a smaller linear spectral chirp, a smaller enhancement of the dissociation probability would have been obtained. Thus, the dissociation probability is about $10 \%$ smaller for a pure linear positive chirp compared to pulse I and about $30 \%$ smaller for a pure linear negative chirp compared to pulse II.

The experimental observation of these coherently controlled transient fragment distributions should be feasible. The detection of, for example, a transient dissociation probability was already demonstrated in the first papers on the femtochemistry of NaI. ${ }^{17}$ A detection scheme for transient momentum distributions has also been discussed. ${ }^{23}$ Figure 4 shows the momentum distributions at two different times with the two kinds of phase defined in Eqs. (17) and (18). The origin of the characteristic structures in these distributions has been discussed previously. ${ }^{24-26}$ It is very interesting to note that the phase-only shaped pulses can change the relative importance of the various momentum components, for example, the low-energy components are enhanced with the first kind of phase function, and the high-energy components are enhanced with the second one. This effect is clearly observed even after 3 ps corresponding to about 3 vibrational periods of the quasi-bound $(\mathrm{NaI}) *$ complex.

Again, it should be noted that the 600 fs FWHM of the optimized pulse is shorter than the 740 fs of the linearly chirped pulse in Ref. 15, but the effect of phase on the transient momentum distributions with the optimized laser pulse is clearly larger than that with the linearly chirped pulses. Thus, comparing, e.g., pulse I in Fig. 4(a) with the positively chirped pulse in Fig. 6(a) of Ref. 15, we observe that the suppression of high momenta is stronger with the optimized pulse, implying that higher order phase modulation is also important for the optimization.

\section{CONCLUSIONS}

In this work we presented-to the best of our knowledge-the first numerical implementation of phaseonly shaped laser pulses within the quantum optimal control theory of laser-molecule interaction. Furthermore, we implemented a constraint which leads to symmetric pulses. We applied this approach to the weak-field photofragmentation of $\mathrm{NaI}$, which is an indirect fragmentation via a quasi-bound complex $(\mathrm{NaI})^{*}$. We showed that the transient dissociation probability as well as the relative momentum distribution of the fragments $\mathrm{Na}$ and I, can be substantially modified after an optimization of the phases associated with the various frequency components of the laser pulse. As expected, the effect is larger than the one observed in our previous work ${ }^{15}$ where only simple phase functions leading to linearly chirped pulses were employed. As in our previous study, ${ }^{15}$ it should be emphasized that phase-only control in the weak-field limit is restricted to transient fragment distribution. That is, no control is observed in the long-time limit where the fragmentation is completed.

\section{ACKNOWLEDGMENTS}

The Technical University of Denmark is acknowledged for a scholarship under the H. C. Ørsted postdoc programme.

${ }^{1}$ A. M. Weiner, Opt. Commun. 284, 3669 (2011).

${ }^{2}$ C. Brif, R. Chakrabarti, and H. Rabitz, New J. Phys. 12, 075008 (2010).

${ }^{3} \mathrm{M}$. Shapiro and P. Brumer, Principles of Quantum Control of Molecular Processes (Wiley, New York, 2003).

${ }^{4}$ N. E. Henriksen, Chem. Soc. Rev. 31, 37 (2002).

5 J. Werschnik and E. K. U. Gross, J. Phys. B 40, R175 (2007).

${ }^{6}$ A. Assion, T. Baumert, M. Bergt, T. Brixner, B. Kiefer, V. Seyfried, M. Strehle, and G. Gerber, Science 282, 919 (1998).

${ }^{7}$ E. Hertz, A. Rouzée, S. Guérin, B. Lavorel, and O. Faucher, Phys. Rev. A 75, 031403 (2007)

${ }^{8}$ P. Brumer and M. Shapiro, Chem. Phys. Lett. 126, 541 (1986).

${ }^{9}$ P. Brumer and M. Shapiro, Chem. Phys. 139, 221 (1989). 
${ }^{10}$ M. Shapiro and P. Brumer, J. Phys. Chem. A 105, 2897 (2001).

${ }^{11}$ N. E. Henriksen, Adv. Chem. Phys. 91, 433 (1995).

${ }^{12}$ M. Spanner, C. A. Arango, and P. Brumer, J. Chem. Phys. 133, 151101 (2010).

${ }^{13}$ J. L. Krause, R. M. Whitnell, K. R. Wilson, Y.-J. Yan, and S. Mukamel, J. Chem. Phys. 99, 6562 (1993).

${ }^{14}$ A. K. Tiwari, K. B. Møller, and N. E. Henriksen, Chem. Phys. Lett. 450, 6 (2007).

${ }^{15}$ C. C. Shu and N. E. Henriksen, J. Chem. Phys. 134, 164308 (2011).

${ }^{16}$ T. Cheng and A. Brown, J. Chem. Phys. 124, 034111 (2006).

${ }^{17}$ T. S. Rose, M. J. Rosker, and A. H. Zewail, J. Chem. Phys. 91, 7415 (1989).

${ }^{18}$ V. Engel and H. Metiu, J. Chem. Phys. 90, 6116 (1989).
${ }^{19}$ G. H. Peslherbe, R. Bianco, J. T. Hynes, and B. M. Ladanyi, J. Chem. Soc., Faraday Trans. 93, 977 (1997)

${ }^{20}$ G. Katz, M. A. Ratner, and R. Kosloff, New. J. Phys. 12, 015003 (2010).

${ }^{21}$ J. Degert, C. Meier, B. Girard, and M. J. J. Vrakking, Eur. Phys. J. D 14, 257 (2001).

${ }^{22}$ W.-S. Zhu and H. Rabitz, J. Chem. Phys. 109, 385 (1998).

${ }^{23}$ A. V. Baklanov, L. M. C. Janssen, D. H. Parker, L. Poisson, B. Soep, J. M. Mestdagh, and O. Gobert, J. Chem. Phys. 129, 214306 (2008).

${ }^{24}$ S. Chapman and M. S. Child, J. Phys. Chem. 95, 578 (1991).

${ }^{25}$ C. Meier, V. Engel, and J. S. Briggs, J. Chem. Phys. 95, 7337 (1991).

${ }^{26}$ N. E. Henriksen, J. Chem. Phys. 132, 234311 (2010). 